Ada Ávila Assunção ${ }^{1}$

Adriano Marçal Pimenta ${ }^{2}$

\section{A exposição à vibração e a hipertensão arterial em trabalhadores do transporte coletivo metropolitano}

\author{
Exposure to vibration and high blood pressure among \\ metropolitan public transport workers
}

\footnotetext{
${ }^{1}$ Universidade Federal de Minas Gerais, Departamento de Medicina Preventiva e Social. Belo Horizonte, MG, Brasil.

${ }^{2}$ Universidade Federal de Minas Gerais, Departamento de Enfermagem Materno-Infantil e Saúde Pública. Belo Horizonte, MG, Brasil.
}

Contato:

Ada Ávila Assunção

E-mail:

adavila@medicina.ufmg.br

O estudo não foi baseado em tese e não foi apresentado em eventos científicos.

Estudo apoiado pelo Conselho Nacional de Desenvolvimento Científico e Tecnológico (CNPq- Processo 458922/2014-5).

Os autores declaram não haver conflitos de interesses.

\section{Resumo}

Objetivo: esclarecer possíveis associações entre a exposição à vibração no trabalho em ônibus da região metropolitana de Belo Horizonte-MG e o autorrelato de hipertensão arterial (HA). Métodos: questionário eletrônico preenchido em entrevista com motoristas $(n=853)$ e cobradores $(n=754)$, em pontos de parada das empresas, em 2012. Amostra estimada considerando-se as quotas de trabalhadores (universo amostral $=17.470$ motoristas e cobradores) distribuídos nas cidades de Belo Horizonte, Betim e Contagem. A força das associações foi medida pelas razões de prevalência e seus intervalos de confiança de 95\%. Após análise bivariada, a associação independente entre vibração e HA foi avaliada por modelos múltiplos de regressão de Poisson com variâncias robustas, ajustada por potenciais fatores de confusão. Resultados: quanto à resposta sobre exposição à vibração: nunca/raramente $=39,7 \%$ (nunca $=33,5 \%$ e raramente $=6,2 \%$ ); às vezes $=21 \%$; quase sempre/sempre $=39,3 \%$ (quase sempre $=5,9 \%$; sempre $=33,4 \%$ ). HA foi autorrelatada por $14,3 \%$, sendo $33 \%$ mais frequente no grupo que informou sentir o corpo vibrar quase sempre/sempre, quando comparado ao grupo que informou nunca ou raramente (RP: 1,33; IC 95\%: 1,02-1,73). Conclusão: a associação entre vibração e hipertensão salienta a relevância de se considerar a exposição ocupacional na abordagem das morbidades cardiovasculares em adultos.

Palavras-chave: vibração; hipertensão; motoristas de ônibus; saúde ocupacional.

\begin{abstract}
Objective: explain the possible associations between occupational exposure to vibration on buses in the metropolitan region of Belo Horizonte, $M G$, and self-reporting of high blood pressure (HBP). Methods: electronic forms completed during interviews with drivers $(n=853)$ and conductors $(n=754)$ at the bus company yards in 2012. The sample size was estimated taking into consideration the proportions of workers (overall total for sampling $=17,470$ drivers and conductors) distributed in the municipalities Belo Horizonte, Betim and Contagem. The association power was measured by prevalence ratios and their 95\% confidence intervals. After bivariate analysis, the independent association between vibration and HBP was evaluated using multiple Poisson regression models with robust variance, adjusted for potential confounding factors. Results: responses regarding vibration: never $/$ rarely $=39.7 \%$ (never $=33.5 \%$ and rarely $=6.2 \%$, sometimes $=21 \%$; almost always /always $=39.3 \%$ (almost always $=5.9 \%$; always $=33.4 \%$ ). HBP was self-reported by $14.3 \%$ and was $33 \%$ more frequent in the group that stated to feel their body vibrate almost always/always, in comparison with the group that stated never or rarely (PR: 1.33; 95\% CI: 1.02-1.73). Conclusion: the association between vibration and hypertension highlights the importance of considering the occupational exposure in addressing cardiovascular morbidity in adults.
\end{abstract}

Keywords: vibration; hypertension; bus driver; occupational health. 


\section{Introdução}

O trabalhador encontra-se exposto à vibração quando um segmento ou todo o seu corpo transmite um movimento oscilatório - em torno de um ponto fixo produzido por uma estrutura sólida (piso, assento, cabo ou manopla de uma ferramenta ou de uma máquina) (CANADIAN CENTRE FOR OCCUPATIONAL HEALTH AND SAFETY, 2012).

A vibração é caracterizada pelas variáveis velocidade, aceleração e amplitude de deslocamento das partículas que o movimento produz na estrutura corporal. Cada segmento do corpo humano responde de forma específica à vibração. Por meio de instrumentos específicos, como os filtros de ponderação dos vibrômetros, é possível avaliar de maneira integrada os resultados das distintas variáveis, de acordo com os eixos de direção X, Y ou Z (frente e costas, lateral e longitudinal, respectivamente) dos movimentos no corpo inteiro ou em um de seus segmentos (membros superiores, por exemplo) (CANADIAN CENTRE FOR OCCUPATIONAL HEALTH AND SAFETY, 2012).

Síndrome de Vibração diz respeito a um conjunto de sintomas vasculares, neurológicos e musculoesqueléticos. Sensação de dormência ou formigamento, e episódios vasculares nas mãos e dedos (Síndrome dos Dedos Brancos ou Fenômeno de Raynauld) estão associados às operações com ferramentas vibratórias durante o trabalho (BUHAUG; MOEN; IRGENS, 2014).

Há indícios de que vibração localizada em membros superiores esteja relacionada à disfunção no sistema nervoso autônomo (VIROKANNAS; TOLONEN, 1995). Trabalhadores expostos à vibração de corpo inteiro têm mais chance de aumento da pressão arterial do que os controles (BJÖR et al., 2006; IDZIOR-WALUS, 1987).

Estudos experimentais confirmam que a exposição à vibração pode elevar a pressão arterial. Estudos epidemiológicos sobre fatores de risco para infarto agudo do miocárdio identificaram elevada incidência de hipertensão arterial (HA), quando compararam controles e casos expostos a vibração. Tais resultados justificam as indagações quanto à exposição à vibração e às morbidades cardiovasculares em trabalhadores do transporte, sabidamente mais expostos a esse agente, juntamente com os metalúrgicos e trabalhadores da indústria extrativista vegetal, quando comparados aos outros grupos ocupacionais (BJÖR et al., 2006).

A pressão arterial aumentada é considerada um fator de risco linear, contínuo e independente para as doenças cardiovasculares. Tais doenças são responsáveis por mais de um terço do total de óbitos no país, com destaque para as enfermidades isquêmicas do coração e os acidentes vasculares encefálicos (WILLIAMS, 2009). Os motoristas, em geral, independentemente do tipo de veículo que conduzem, são vulneráveis a tais enfermidades (GUSTAVSSON et al., 1996).

No Brasil, apesar do reconhecimento da Síndrome da Vibração como doença relacionada ao trabalho (BRASIL, 2001), não havia, até o presente momento, estudos que enfatizassem resultados obtidos por autorrelatos sobre exposição a tal agente entre os trabalhadores dos ônibus urbanos e com enfoque na HA. Tal lacuna reflete negativamente tanto na formação dos profissionais de saúde do trabalhador quanto na elaboração de programas de prevenção e diagnóstico precoce.

O presente estudo objetivou analisar a relação entre percepção de vibração localizada ou de corpo inteiro, e autorrelato de HA, em uma amostra de trabalhadores do transporte coletivo de ônibus na região metropolitana de Belo Horizonte (RMBH), Minas Gerais.

\section{Métodos}

\section{Tipo, população e amostra}

O presente estudo, de caráter transversal, integra a pesquisa "Condições de saúde e trabalho de motoristas e cobradores do transporte coletivo urbano das cidades de Belo Horizonte, Betim e Contagem, Minas Gerais, Brasil” (ASSUNÇÃO, 2013).

A população elegível no total das três cidades investigadas correspondeu a 17.470 trabalhadores (Belo Horizonte $=6.500$ motoristas e 6.750 cobradores; Betim = 696 motoristas e 524 cobradores; Contagem $=1.800$ motoristas e 1.200 cobradores) (EMPRESA DE TRANSPORTES E TRÂNSITO DE BELO HORIZONTE, 2009).

Para o cálculo amostral, foram considerados os seguintes parâmetros: $22 \%$ de prevalência do desfecho HA (BENVEGNÚ et al., 2008); margem de erro de 3\% (bicaudal); nível de confiança de 95\%, e previsão de perdas de $10 \%$, totalizando 773 participantes. Além de estimar a proporção de HA entre os participantes, o estudo objetivou avaliar a associação independente da vibração do corpo com este desfecho. Para tal, foram considerados os seguintes parâmetros para o cálculo amostral: poder estatístico de $80 \%$; nível de confiança de 95\%; razão de prevalência de 2,00; proporção expostos/não expostos à vibração do corpo de 2 para 1; previsão de perdas de 10\%, e acréscimo de $15 \%$ para o ajuste de fatores de confusão, totalizando 566 participantes.

Por se tratar de um inquérito mais abrangente, no qual outros desfechos de menor prevalência também foram estudados, ao final, foram entrevistados, entre abril e junho de 2012, 1.607 indivíduos, sendo 
853 motoristas e 754 cobradores, distribuídos da seguinte forma: Belo Horizonte $-72 \%$ de motoristas e $80 \%$ de cobradores, Contagem - 20\% de motoristas e $14 \%$ de cobradores, e Betim - 8\% de motoristas e $6 \%$ de cobradores. Portanto, o número de respondentes ultrapassou em $107 \%$ e $184 \%$ as amostras calculadas quando se tomam, respectivamente, o estudo da frequência da HA e o estudo sobre a associação independente entre a vibração do corpo e este desfecho. Dessa forma, aumentou-se o poder estatístico dos testes utilizados.

\section{Coleta de dados}

A coleta de dados foi realizada face a face, com auxílio de netbooks nos turnos manhã e tarde. Elaborou-se um software exclusivo para os fins da pesquisa, tendo em vista os objetivos de preenchimento do questionário digital pelo entrevistador e o processamento on-line dos dados.

Dados da literatura e das entrevistas prévias com representantes sindicais e trabalhadores orientaram a elaboração do instrumento de coleta de dados, que contava com 82 questões a respeito dos seguintes quesitos: demográficos (sexo, idade, cor de pele, estado civil); socioeconômicos (escolaridade, renda familiar); condições de trabalho (cargo, turno de trabalho, horas de trabalho, tempo de trabalho na empresa, férias, jornada de trabalho); ambiente de trabalho interno (ruído, vibração, iluminação, temperatura) e externo (trânsito, ruído); ergonomia; equipamentos de proteção individual; hábitos e estilo de vida (tabagismo, etilismo, atividade física, atividades socioculturais); condições de saúde (uso de medicamentos e doenças diagnosticadas); antropometria (peso, estatura); violência (acidentes de trânsito, conflitos com passageiros), e qualidade de vida.

Os instrumentos e procedimentos da pesquisa foram previamente testados na etapa-piloto (30 participantes). Realizaram-se oficinas específicas dirigidas pelos coordenadores da pesquisa para treinar 22 entrevistadores recrutados em cursos da Universidade Federal de Minas Gerais. A confiabilidade da entrevista foi aferida por meio da reaplicação para o mesmo respondente - $12 \%$ do total dos participantes - de algumas perguntas selecionadas do questionário original.

As entrevistas foram realizadas em quatro estações ônibus-metrô de Belo Horizonte e em 35 paradas de ônibus das três cidades. O contato dos entrevistadores com os sujeitos foi de forma livre e ocasional. Tais paradas de ônibus, também chamadas de estações de descanso, foram selecionadas porque concentram a maioria dos ônibus, das viagens e dos trabalhadores registrados. Estas são destinadas à parada de ônibus, quando os profissionais fazem uma pausa depois de uma viagem de 60 a 90 minutos. Nas quatro estações selecionadas, circulam, em média, 80\% dos passageiros que utilizam o serviço (EMPRESA DE TRANSPORTES E TRÂNSITO DE BELO HORIZONTE, 2009).

Realizou-se ampla divulgação da pesquisa por meio de uma rádio em programa dirigido especificamente aos trabalhadores do transporte coletivo urbano da referida área. Além disso, foram distribuídos cartazes e folhetos aos gestores e trabalhadores antes do início do trabalho de campo.

\section{Variável de exposição}

A variável de exposição 'vibração do corpo' foi elaborada com base na resposta à seguinte pergunta do questionário: 'Durante o seu trabalho, você sente o seu corpo vibrar?'. As opções de resposta disponíveis eram: nunca; raramente; às vezes; quase sempre; sempre. Para a análise dos dados, esta variável foi recategorizada em nunca/raramente; às vezes; quase sempre/sempre.

\section{Covariáveis}

Foram examinadas as seguintes variáveis, que apresentavam potencialidade para "confundir" a relação entre vibração do corpo e HA: sexo (masculino, feminino); idade (18-29 anos, 30-39 anos, 40-49 anos, 50-59 anos, 60 e mais anos); renda familiar (até 2 salários mínimos, 2 a 4 salários mínimos, 4 ou mais salários mínimos - o salário mínimo à época era de $\mathrm{R} \$$ 622,00); tabagismo (Considerando como fumante quem já fumou pelo menos 100 cigarros, ou 5 maços, você se classifica como: não fumante, ex-fumante, fumante atual); atividade física (Com que frequência você realiza atividades físicas? nunca, 1 vez por semana, 2 vezes por semana, 3 vezes por semana, 4 ou mais vezes por semana); atividades socioculturais (Você participa de atividades culturais (cinema, teatro, exposição)? não, sim); índice de massa corporal - IMC - calculado pela seguinte equação: peso (kg)/estatura $(\mathrm{m})^{2},<25$ (eutrófico), 25-29,9 (sobrepeso), $\geq 30$ (obeso) (WORLD HEALTH ORGANIZATION, 1995) - tanto o peso quanto a estatura foram autorreferidos pelo participante; temperatura dentro do ônibus (Durante o seu trabalho, como você percebe a temperatura dentro do ônibus? tolerável, incomoda pouco, incomoda muito, insuportável); iluminação dentro do ônibus (Durante o seu trabalho, como você percebe a iluminação dentro do ônibus? boa, regular, ruim, muito ruim); ruído dentro do ônibus (Em geral, o ruído originado dentro do ônibus é: desprezível, razoável, elevado, insuportável). 


\section{Variável de desfecho}

A variável de desfecho, HA, foi elaborada com base nas respostas a duas perguntas do questionário: (1) Você possui diagnóstico médico de pressão alta (hipertensão arterial)? (2) Atualmente, você está fazendo uso de medicamento prescrito por médico para pressão alta (hipertensão arterial)? Para ambas as perguntas, as opções de resposta eram: não, sim.

Foi considerado hipertenso, o respondente que disse ‘sim' a pelo menos uma das duas questões. Os níveis pressóricos foram considerados dentro dos padrões de normalidade quando o participante respondeu negativamente às duas questões.

\section{Análise estatística}

A caracterização da amostra foi realizada por meio do cálculo das frequências absolutas e relativas das variáveis demográficas (sexo e idade), socioeconômicas (renda familiar), estilo de vida (tabagismo, atividade física, atividades socioculturais), antropométricas (IMC) e condições do ambiente interno do ônibus (temperatura, ruído e iluminação), segundo as categorias da variável de exposição (vibração do corpo). As diferenças estatísticas foram avaliadas usando o Teste de Qui-quadrado de Pearson.

A análise bivariada foi conduzida para avaliar a associação bruta da vibração do corpo e de cada covariável de interesse com a HA a partir do Teste de Qui-quadrado de Pearson. Na ocasião em que a covariável tinha três ou mais categorias, foi calculado o $p$ de tendência linear. Ademais, foram estimadas razões de prevalência (RP) e seus respectivos intervalos de confiança de 95\% (IC 95\%) das associações avaliadas por meio da Técnica de Regressão de Poisson com variâncias robustas.

As variáveis, cujas associações com a HA mostraram nível de significância estatística inferior a 20\% $(p<0,20)$ na análise bivariada, foram utilizadas para o ajuste multivariado dos dados por meio da Técnica de Regressão de Poisson com variâncias robustas, sendo a hipótese principal pesquisada a associação entre a vibração do corpo e a HA. Assim, a RP e seu respectivo IC 95\% foram calculados tendo como referência a categoria nunca/raramente da variável vibração do corpo.

No modelo 1, foram consideradas como variáveis de ajuste o sexo e a idade. Para o modelo 2, consideraram-se as variáveis do modelo $1 \mathrm{e}$, adicionalmente, o tabagismo, a realização de atividades socioculturais e o IMC. Por fim, no modelo 3, consideraram-se as variáveis do modelo 2 e, adicionalmente, a percepção da temperatura interna do ônibus. Todos os modelos foram rodados novamente, considerando-se a variável de exposição na sua forma original, ou seja, com cinco categorias (nunca, raramente, às vezes, quase sempre e sempre). Em todas as análises, o nível de significância estatística foi fixado em $5 \%(p<0,05)$.

\section{Aspectos éticos}

A pesquisa foi aprovada pelo Comitê de Ética e Pesquisa em Seres Humanos da Universidade Federal de Minas Gerais (Parecer n. ${ }^{\circ} \mathrm{CAAE}$ - 02705012.4.0000.5149). Todos os participantes assinaram o Termo de Consentimento Livre e Esclarecido.

\section{Resultados}

No que diz respeito à variável de exposição, o relato dos participantes quanto à percepção de vibração foi assim distribuído: nunca/raramente $=39,7 \%$ (nunca $=33,5 \%$ e raramente $=6,2 \%$ ); às vezes $=21,0 \%$; quase sempre/sempre $=39,3 \%$ (quase sempre $=5,9 \%$ e sempre $=33,4 \%$ ).

A maioria dos profissionais era do sexo masculino, em faixas etárias inferiores a 40 anos, renda familiar de até 4 salários mínimos, informando atividades socioculturais e sedentarismo. Além disso, 15,8\% se autodeclararam como fumantes e 49,2\% informaram peso e estatura compatíveis com sobrepeso/obesidade (Tabela 1).

Foram observadas menores proporções de indivíduos fumantes, com alta renda e menos praticantes de atividades socioculturais no grupo que relatou perceber o corpo vibrar quase sempre/sempre. Este grupo relatou maior proporção de sedentarismo (Tabela 1).

Com relação ao ambiente interno do ônibus, $45,0 \%$ dos sujeitos declararam que a temperatura incomodava muito ou era insuportável, e a maioria referiu trabalhar sob boa iluminação, mas exposta a ruído elevado/insuportável (Tabela 1).

Entre os participantes que sentiam o seu corpo vibrar quase sempre/sempre, foram observadas maiores proporções de exposição a piores condições internas do ônibus, pois 56,6\% relataram alto incômodo devido a temperatura ou temperatura insuportável, $11,5 \%$ consideraram a iluminação ruim ou muito ruim, e $62,4 \%$ descreveram o ruído como elevado ou insuportável (Tabela 1).

No geral, a prevalência de HA foi de 14,3\% e, em nível bivariado, as seguintes características a esta se associaram de forma significativa: 1) positivamente - sexo, idade, IMC, percepção da temperatura interna do ônibus; 2) negativamente - realização de atividades socioculturais (Tabela 2). 
Tabela 1 Distribuição da amostra estudada segundo características sociodemográficas, do estilo de vida, antropométricas e das condições internas do ônibus. Região Metropolitana de Belo Horizonte, Minas Gerais, 2012

\begin{tabular}{|c|c|c|c|c|}
\hline \multirow[b]{2}{*}{ Variáveis } & \multicolumn{3}{|c|}{ Vibração do corpo } & \multirow[b]{2}{*}{ Total } \\
\hline & $\begin{array}{c}\text { Nunca/raramente } \\
n(\%)\end{array}$ & $\begin{array}{c}\text { Às vezes } \\
n(\%)\end{array}$ & $\begin{array}{c}\text { Quase sempre/sempre } \\
n(\%)\end{array}$ & \\
\hline \multicolumn{5}{|l|}{ Sexo } \\
\hline Masculino & $560(87,8)$ & $289(85,5)$ & $551(87,3)$ & $1400(87,1)$ \\
\hline Feminino & $78(12,2)$ & $49(14,5)$ & $80(12,7)$ & $207(12,9)$ \\
\hline \multicolumn{5}{|l|}{ Idade (anos) } \\
\hline $18-29$ & $202(31,7)$ & $120(35,5)$ & $233(36,9)$ & $555(34,5)$ \\
\hline $30-39$ & $211(33,1)$ & $108(32,0)$ & $210(33,3)$ & $529(32,9)$ \\
\hline $40-49$ & $150(23,5)$ & $77(22,8)$ & $140(22,2)$ & $367(22,8)$ \\
\hline $50-59$ & $58(9,1)$ & $29(8,6)$ & $42(6,7)$ & $129(8,0)$ \\
\hline 60 ou mais & $17(2,7)$ & $4(1,2)$ & $6(1,0)$ & $27(1,7)$ \\
\hline \multicolumn{5}{|l|}{ Renda familiar (salários mínimos)* } \\
\hline Até 2 & $120(18,8)$ & $93(27,5)$ & $169(26,8)$ & $382(23,8)$ \\
\hline $2-4$ & $305(47,8)$ & $138(40,8)$ & $278(44,1)$ & $721(44,9)$ \\
\hline 4 ou mais & $213(33,4)$ & $107(31,7)$ & $184(29,2)$ & $504(31,4)$ \\
\hline \multicolumn{5}{|l|}{ Tabagismo* } \\
\hline Não fumante & $442(69,3)$ & $222(65,7)$ & $450(71,3)$ & $1114(69,3)$ \\
\hline Ex-fumante & $92(14,4)$ & $47(13,9)$ & $100(15,9)$ & $239(14,9)$ \\
\hline Fumante atual & $104(16,3)$ & $69(20,4)$ & $81(12,8)$ & $254(15,8)$ \\
\hline \multicolumn{5}{|l|}{ Atividade física* } \\
\hline Nunca & $299(46,9)$ & $177(52,4)$ & $342(54,2)$ & $818(50,9)$ \\
\hline 1 a 2 vezes por semana & $162(25,4)$ & $89(26,3)$ & $148(23,5)$ & $399(24,8)$ \\
\hline 3 ou mais vezes por semana & $177(27,7)$ & $72(21,3)$ & $141(22,4)$ & $390(24,3)$ \\
\hline \multicolumn{5}{|l|}{ Atividades socioculturais* } \\
\hline Não & $183(28,7)$ & $107(31,7)$ & $229(36,3)$ & $519(32,3)$ \\
\hline Sim & $455(71,3)$ & $231(68,3)$ & $402(63,7)$ & $1088(67,7)$ \\
\hline \multicolumn{5}{|l|}{ Índice de Massa Corporal (kg/m2) } \\
\hline$<25$ & $328(51,4)$ & $175(52,8)$ & $313(49,6)$ & $816(50,8)$ \\
\hline $25-29,9$ & $221(34,6)$ & $114(33,7)$ & $223(35,3)$ & $558(34,7)$ \\
\hline$\geq 30$ & $89(14,0)$ & $49(14,5)$ & $95(15,1)$ & $233(14,5)$ \\
\hline \multicolumn{5}{|l|}{ Temperatura dentro do ônibus* } \\
\hline Tolerável & $234(36,7)$ & $74(21,9)$ & $117(18,5)$ & $425(26,5)$ \\
\hline Incomoda pouco & $192(30,1)$ & $110(32,5)$ & $157(24,9)$ & $459(28,6)$ \\
\hline Incomoda muito & $169(26,5)$ & $120(35,5)$ & $270(42,8)$ & $559(34,8)$ \\
\hline Insuportável & $43(6,7)$ & $34(10,1)$ & $87(13,8)$ & $164(10,2)$ \\
\hline \multicolumn{5}{|l|}{ Iluminação dentro do ônibus* } \\
\hline Boa & $483(75,7)$ & $227(67,2)$ & $366(58,0)$ & $1076(67,0)$ \\
\hline Regular & $125(19,6)$ & $83(24,6)$ & $192(30,4)$ & $400(24,9)$ \\
\hline Ruim & $26(4,1)$ & $23(6,8)$ & $52(8,2)$ & $101(6,3)$ \\
\hline Muito ruim & $4(0,6)$ & $5(1,5)$ & $21(3,3)$ & $30(1,9)$ \\
\hline \multicolumn{5}{|l|}{ Barulho dentro do ônibus* } \\
\hline Desprezível & $55(8,6)$ & $22(6,5)$ & $42(6,7)$ & $119(7,4)$ \\
\hline Razoável & $341(53,5)$ & $134(39,6)$ & $195(30,1)$ & $670(41,7)$ \\
\hline Elevado & $161(25,2)$ & $96(28,4)$ & $221(35,0)$ & $478(29,7)$ \\
\hline Insuportável & $81(12,7)$ & $86(25,4)$ & $173(27,4)$ & $340(21,2)$ \\
\hline
\end{tabular}

"p $<0,05$ para o Teste de Qui-quadrado de Pearson. 
Tabela 2 Associação entre vibração do corpo e covariáveis de interesse com a hipertensão arterial entre motoristas e cobradores de ônibus. Região Metropolitana de Belo Horizonte, Minas Gerais, 2012

\begin{tabular}{|c|c|c|c|c|c|}
\hline \multirow{2}{*}{ Variáveis } & \multicolumn{5}{|c|}{ Hipertensão arterial } \\
\hline & $n$ & $\%$ & $R P$ & IC 95\% & $p$ \\
\hline Sexo & & & & & $0,027^{*}$ \\
\hline Masculino & 190 & 13,6 & 1,00 & - & \\
\hline Feminino & 40 & 19,3 & 1,42 & $1,05-1,94$ & \\
\hline Idade (anos) & & & & & $<0,001^{\dagger}$ \\
\hline $18-29$ & 24 & 4,3 & 1,00 & - & \\
\hline $30-39$ & 59 & 11,2 & 2,58 & $1,63-4,08$ & \\
\hline $40-49$ & 84 & 22,9 & 5,29 & $3,43-8,17$ & \\
\hline $50-59$ & 53 & 41,1 & 9,50 & $6,10-14,79$ & \\
\hline 60 ou mais & 10 & 37,0 & 8,56 & $4,57-16,06$ & \\
\hline Renda familiar (salários mínimos) & & & & & $0,596^{\dagger}$ \\
\hline Até 2 & 53 & 13,9 & 1,00 & - & \\
\hline $2-4$ & 101 & 14,0 & 1,01 & $0,74-1,37$ & \\
\hline 4 ou mais & 76 & 15,1 & 1,09 & $0,79-1,50$ & \\
\hline Tabagismo & & & & & $0,051^{\dagger}$ \\
\hline Não fumante & 145 & 13,0 & 1,00 & - & \\
\hline Ex-fumante & 43 & 18,0 & 1,38 & $1,01-1,89$ & \\
\hline Fumante atual & 42 & 16,5 & 1,27 & $0,93-1,74$ & \\
\hline Atividade física & & & & & $0,217^{\dagger}$ \\
\hline Nunca & 130 & 15,9 & 1,00 & - & \\
\hline 1 a 2 vezes por semana & 46 & 11,5 & 0,73 & $0,53-0,99$ & \\
\hline 3 ou mais vezes por semana & 54 & 13,9 & 0,87 & $0,65-1,17$ & \\
\hline Atividades socioculturais & & & & & $0,037^{*}$ \\
\hline Não & 88 & 17,0 & 1,00 & - & \\
\hline Sim & 142 & 13,1 & 0,77 & $0,60-0,98$ & \\
\hline Índice de Massa Corporal (kg/m2) & & & & & $<0,001^{\dagger}$ \\
\hline$<25$ & 81 & 9,9 & 1,00 & - & \\
\hline $25-29,9$ & 91 & 16,3 & 1,64 & $1,24-2,17$ & \\
\hline$\geq 30$ & 58 & 24,9 & 2,51 & $1,85-3,40$ & \\
\hline Temperatura dentro do ônibus & & & & & $0,002^{\dagger}$ \\
\hline Tolerável & 49 & 11,5 & 1,00 & - & \\
\hline Incomoda pouco & 58 & 12,6 & 1,10 & $0,77-1,57$ & \\
\hline Incomoda muito & 89 & 15,9 & 1,38 & $1,00-1,91$ & \\
\hline Insuportável & 37 & 20,7 & 1,80 & $1,21-2,68$ & \\
\hline Iluminação dentro do ônibus & & & & & $0,769^{\dagger}$ \\
\hline Boa & 155 & 14,4 & 1,00 & - & \\
\hline Regular & 55 & 13,8 & 0,95 & $0,72-1,27$ & \\
\hline Ruim & 13 & 12,9 & 0,89 & $0,53-1,52$ & \\
\hline Muito ruim & 7 & 23,3 & 1,62 & $0,83-3,15$ & \\
\hline Barulho dentro do ônibus & & & & & $0,825^{\dagger}$ \\
\hline Desprezível & 14 & 11,8 & 1,00 & - & \\
\hline Razoável & 102 & 15,2 & 1,29 & $0,77-2,18$ & \\
\hline Elevado & 68 & 14,2 & 1,21 & $0,71-2,07$ & \\
\hline Insuportável & 46 & 13,5 & 1,15 & $0,66-2,01$ & \\
\hline Vibração do corpo & & & & & $0,104^{\dagger}$ \\
\hline Nunca/raramente & 78 & 12,2 & 1,00 & - & \\
\hline Às vezes & 55 & 16,3 & 1,33 & $0,97-1,83$ & \\
\hline Quase sempre/sempre & 97 & 15,4 & 1,26 & $0,95-1,66$ & \\
\hline
\end{tabular}

"P-valor do Teste de Qui-quadrado de Pearson.

'P-valor do Teste de Wald para tendência linear. 
Tabela 3 Associação independente entre vibração do corpo e hipertensão arterial entre motoristas e cobradores de ônibus. Região Metropolitana de Belo Horizonte, Minas Gerais, 2012

\begin{tabular}{|c|c|c|c|}
\hline & $R P$ & IC $95 \%$ & $p$ \\
\hline \multicolumn{4}{|l|}{ Modelo $1^{*}$} \\
\hline Vibração do corpo & & & $0,009 \S$ \\
\hline Nunca/raramente & 1,00 & - & - \\
\hline Às vezes & 1,40 & $1,04-1,89$ & 0,028 \\
\hline Quase sempre/sempre & 1,42 & $1,09-1,84$ & 0,010 \\
\hline \multicolumn{4}{|l|}{ Modelo $2^{\dagger}$} \\
\hline Vibração do corpo & & & $0,012^{\S}$ \\
\hline Nunca/raramente & 1,00 & - & - \\
\hline Às vezes & 1,38 & $1,02-1,87$ & 0,035 \\
\hline Quase sempre/sempre & 1,40 & $1,07-1,81$ & 0,013 \\
\hline \multicolumn{4}{|l|}{ Modelo $3^{\ddagger}$} \\
\hline Vibração do corpo & & & $0,049 \S$ \\
\hline Nunca/raramente & 1,00 & - & - \\
\hline Às vezes & 1,33 & $0,98-1,79$ & 0,067 \\
\hline Quase sempre/sempre & 1,30 & $1,01-1,69$ & 0,049 \\
\hline
\end{tabular}

"Modelo 1: Ajustado por sexo e idade.

†Modelo 2: Modelo 1 adicionalmente ajustado por tabagismo, atividades socioculturais e índice de massa corporal.

Modelo 3: Modelo 2 adicionalmente ajustado pela percepção da temperatura interna do ônibus.

${ }_{\S}^{\S}$-valor do Teste de Wald para tendência linear.

Tabela 4 Associação independente entre vibração do corpo (categorias originais da variável) e hipertensão arterial entre motoristas e cobradores de ônibus. Região Metropolitana de Belo Horizonte, Minas Gerais, 2012

\begin{tabular}{|c|c|c|c|}
\hline & $R P$ & IC 95\% & $p$ \\
\hline \multicolumn{4}{|l|}{ Modelo $1^{*}$} \\
\hline Vibração do corpo & & & $0,021^{\S}$ \\
\hline Nunca & 1,00 & - & - \\
\hline Raramente & 0,76 & $0,40-1,45$ & 0,411 \\
\hline Às vezes & 1,35 & $0,99-1,84$ & 0,055 \\
\hline Quase sempre & 1,80 & $1,10-2,94$ & 0,019 \\
\hline Sempre & 1,31 & $0,98-1,73$ & 0,064 \\
\hline \multicolumn{4}{|l|}{ Modelo $2^{\dagger}$} \\
\hline Vibração do corpo & & & $0,026^{\S}$ \\
\hline Nunca & 1,00 & - & - \\
\hline Raramente & 0,77 & $0,40-1,48$ & 0,434 \\
\hline Às vezes & 1,34 & $0,98-1,82$ & 0,065 \\
\hline Quase sempre & 1,77 & $1,08-2,89$ & 0,022 \\
\hline Sempre & 1,29 & $0,97-1,70$ & 0,076 \\
\hline Modelo 3 & & & $0,108^{\S}$ \\
\hline \multicolumn{4}{|l|}{ Vibração do corpo } \\
\hline Nunca & 1,00 & - & - \\
\hline Raramente & 0,72 & $0,38-1,40$ & 0,334 \\
\hline Às vezes & 1,26 & $0,93-1,72$ & 0,137 \\
\hline Quase sempre & 1,68 & $1,03-2,74$ & 0,036 \\
\hline Sempre & 1,17 & $0,89-1,55$ & 0,262 \\
\hline
\end{tabular}

*Modelo 1: Ajustado por sexo e idade.

†Modelo 2: Modelo 1 adicionalmente ajustado por tabagismo, atividades socioculturais e índice de massa corporal.

"Modelo 3: Modelo 2 adicionalmente ajustado pela percepção da temperatura interna do ônibus.

${ }^{\S}$ p-valor do Teste de Wald para tendência linear. 
Ainda que a vibração do corpo não tenha se associado à HA em nível bivariado, esta relação estava provavelmente sofrendo influência das variáveis de confusão. Assim, na análise multivariável dos dados, após o ajuste por sexo e idade (Modelo 1), as percepções da vibração do corpo como 'às vezes' e 'quase sempre/sempre' permaneceram independentemente associadas à HA (Tabela 3). O ajuste adicional por tabagismo, realização de atividades socioculturais e IMC (Modelo 2) diminuiu a força de associação entre ambas as categorias da variável de exposição e HA, porém sem acarretar perda de significância estatística.

O ajuste adicional considerando a percepção da temperatura interna do ônibus (Modelo 3) provocou a perda da associação entre a percepção da vibração do corpo como ‘às vezes' e HA. Mais uma vez, o ajuste reduziu a força de associação entre a percepção da vibração do corpo 'quase sempre/sempre' e HA, mantendo-se, porém, a significância estatística (Tabela 3).

Em todos os modelos, observa-se que o $p$ de tendência linear foi significativo, indicando que quanto mais frequente o trabalhador percebia o seu corpo vibrar maior era a proporção de HA (Tabela 3).

Por fim, todos os modelos foram mais uma vez "rodados", considerando-se a variável de exposição na sua forma original (Tabela 4). Ainda assim, a percepção da vibração do corpo ‘quase sempre' durante o trabalho permaneceu independentemente associada à HA, mesmo após o ajuste por todas as potenciais variáveis de confusão. Houve tendência linear para a associação da percepção do corpo vibrar e HA nos modelos 1 e 2, mas esta perdeu significância estatística no modelo, após o ajuste adicional da associação pela percepção da temperatura interna do ônibus (Tabela 4).

\section{Discussão}

A exposição à vibração foi avaliada por meio dos autorrelatos de trabalhadores dos ônibus da região metropolitana de Belo Horizonte, Minas Gerais, com o intuito de examinar a associação com HA autorrelatada. Quanto a esta, os resultados obtidos foram inferiores ao inquérito populacional, que identificou 20,8\% entre os adultos da cidade de Belo Horizonte (LIMA-COSTA; TURCI; MACINKO, 2012). Em grupos de motoristas, a prevalência tem se mostrado superior a 20\% (BENVEGNÚ et al., 2008; COSTA et al., 2003). Diferenças nos procedimentos e técnicas de coleta dos dados podem explicar, ao menos parcialmente, as discrepâncias.

Nos estudos citados (com exceção do inquérito populacional), incluiu-se a aferição direta da pressão arterial obtida por leituras circunscritas. Ainda que aceitável concluir sobre a HA baseando-se somente em uma leitura, não está descartada a possibilidade de superestimação das taxas obtidas naqueles estudos que identificaram mais de 20\% de HA (SOCIEDADE BRASILEIRA DE HIPERTENSÃO, 2010). É possível ainda que, ao incluir o diagnóstico médico na pergunta que deu origem à variável, tenha sido descartado um contingente de trabalhadores hipertensos sem acesso aos ambulatórios especializados, explicando menos relatos de HA no presente estudo, se comparado à literatura.

Consideradas as limitações de cunho tanto metodológico (inquérito transversal) quanto paradigmático (multicausalidade), em se tratando de alterações cardiovasculares, este trabalho traz uma contribuição para os programas de promoção e vigilância à saúde dos trabalhadores. A HA foi 33\% mais frequente no grupo que relatou sentir o corpo vibrar 'quase sempre/sempre' quando comparado àqueles que 'nunca ou raramente' estavam submetidos a tais condições de trabalho. Tal resultado é consistente (LEWIS; JOHNSON, 2012), indicando a necessidade de elaboração de medidas para prevenir o desenvolvimento da HA e suprimir suas consequências - infarto agudo do miocárdio, por exemplo (BJÖR et al., 2006; BIGERT et al., 2003). Ademais, o relato de exposição permanente (quase sempre) à vibração do corpo durante o trabalho também permaneceu independentemente associado à HA, mesmo após o ajuste por todas as potenciais variáveis de confusão (RP: 1,68; IC 95\%: 1,03-2,74).

Os trabalhadores dos ônibus são frequentemente vítimas de condições de trabalho inadequadas e, consequentemente, de problemas de saúde associados à sua atividade laboral. O motorista e o seu auxiliar dividem, em seu cotidiano de trabalho, dois ambientes: a) o interno, cujos equipamentos, sistemas de suspensão, posição do motor, estado de conservação das engrenagens etc. são fontes de ruído e de vibração (THAMSUWAN et al., 2013), e b) o externo, cujo estado de conservação da pista e condições do trânsito provocam ou agravam os efeitos da vibração originada em outras fontes (BATTISTON; CRUZ; HOFFMANN, 2006; LEWIS; JOHNSON, 2012).

Apesar de a Norma 15.570 da Associação Brasileira de Normas Técnicas (2011) especificar as características e condições para a fabricação de veículos para transporte coletivo urbano de passageiros, observou-se que a maioria da frota na RMBH está defasada nos referidos quesitos (ASSUNÇÃO, 2013).

A circulação dos ônibus nas regiões metropolitanas se dá tanto em vias quilométricas, interurbanas, por exemplo, quanto em vias locais, que ligam subúrbios e área central das cidades. A interação entre o tipo do ônibus (engrenagens e estrutura/conformação do piso 
e do assento do motorista e do cobrador) e o tipo de via de circulação pode gerar a vibração.

Uma possível explicação para os resultados que indicam mais relatos de exposição à vibração seria a sobreposição de efeitos negativos sobre o organismo quando são precários tanto os sistemas de suspensão dos veículos quanto o tipo de assento e o estado de conservação da estrada ou via na qual o ônibus circula. A exposição à vibração em ônibus de piso mais alto em relação ao solo é menor quando ele circula nas rodovias ou nas ruas bem pavimentadas da cidade do que quando o ônibus circula em vias mal pavimentadas ou com quebra-molas. Nessas situações inadequadas, o corpo do motorista, ao permanecer muito distante do solo (no caso dos ônibus de piso mais alto), fica exposto à exacerbação da transmissão da vibração em suas estruturas. Sendo assim, aumenta-se o movimento do corpo no plano transverso tanto no eixo lateral (direito-esquerdo) quanto no eixo frente e atrás (anteroposterior) (THAMSUWAN et al., 2013).

No presente estudo, não foi possível examinar os relatos de acordo com o tipo de ônibus ou das características das vias de circulação, por várias razões: os trabalhadores estão inseridos em mais de um tipo de empresa; eles manejam mais de um tipo de veículo, na medida em que cada empresa incorpora novos carros sem necessariamente substituir os antigos; nem sempre são mantidas as mesmas linhas de circulação para os mesmos profissionais (ASSUNÇÃO, 2013).

A abordagem da exposição e do desfecho por meio de autorrelatos apresenta seus limites. Por que empregar tal estratégia de coleta de dados diante da exequibilidade de se examinar tanto a condição ambiental quanto a situação mórbida por meio de instrumentos e métodos diretos de mensuração? Aqui vale uma explicação. A relevância deste estudo foi trazer à tona indícios quanto às condições laborais, as quais, raramente, integram as perguntas dos inquéritos epidemiológicos. O insignificante interesse dos inquéritos populacionais em destacar os fatores ocupacionais desse tipo talvez explique a escassez de informações, com reflexos sobre os programas de promoção e vigilância em saúde dos trabalhadores. Paradoxalmente, enfoques ocupacionais já evidenciaram aumento do risco de infarto agudo do miocárdio entre motoristas de ônibus, os quais, entre outros fatores, estavam expostos à vibração durante o trabalho (ALFREDSSON; HAMMAR; HOGSTEDT, 1993; GUSTAVSSON et al., 1996).

A estratégia de conduzir inquéritos em grupos ocupacionais permite examinar a hipótese sobre a contribuição do trabalho nos fatos de saúde. Os grupos ocupacionais são frações da população geral, que apresentam em comum o vínculo de emprego e suas circunstâncias, denominadas condições de trabalho.
Os inquéritos ocupacionais têm a vantagem de, ao enfatizarem exposições específicas àquele trabalho - por exemplo, tarefas extenuantes ou exposição ao ruído dentro e fora do ônibus e a vibração de corpo inteiro -, ampliar o leque de hipóteses sobre os sintomas autorrelatados ou morbidades confirmadas.

Preocupações nesse âmbito não eliminam as insuficiências de delineamentos transversais e colhidos por autorrelatos, pois são esperados vieses de memória e problemas de sensibilidade e de confiabilidade, dado o espectro multideterminado das condições que levam os respondentes a afirmarem "sim" tanto para a pergunta sobre a vibração quanto para a pergunta sobre HA, entre outros. Assim, há limitações na interpretação dos resultados. Alertase sobre a provável subestimação dos resultados apresentados por pelo menos três motivos: o efeito do respondente voluntário (FROOM et al., 1999), o formato da coleta face a face e o efeito do trabalhador sadio (MONSON, 1986). Quanto a este, sabe-se que os sujeitos mais gravemente acometidos pelos efeitos da exposição à vibração podem ter sido excluídos do emprego. Em relação à entrevista face a face, provavelmente, as respostas foram influenciadas por um comportamento que adere às normas de conveniência social. Se subestimados, os resultados são ainda mais preocupantes. Apesar dos limites, a alta taxa de resposta é encorajadora para amparar as interpretações que foram apresentadas. Consideram-se úteis os resultados apresentados para os planos de promoção e de vigilância à saúde dos trabalhadores.

Ao conhecer a magnitude e a força das associações, os tomadores de decisão poderão obter argumentos para a transformação das condições que produzem a exposição à vibração: mudanças administrativas ou organizacionais e medidas de engenharia. Quanto às últimas, sabe-se que a vibração tem origem na concepção inadequada dos assentos e do veículo como um todo. Além da implantação de assentos ergonômicos, está indicada a manutenção periódica dos mecanismos de suspensão. A Norma Técnica 15.570 (ASSOCIAÇÃO BRASILEIRA DE NORMAS TÉCNICAS, 2011) é bastante completa no tocante aos fatores de exposição à vibração.

\section{Contribuições de autoria}

Assunção, A. A.: elaborou o projeto e coordenou a pesquisa de campo, buscou a bibliografia de base e estruturou o artigo. Pimenta, A. M.: foi responsável pela concepção do artigo e da análise, e pela interpretação dos dados. Ambos os autores participaram da revisão crítica e da redação final do texto. 


\section{Referências}

ALFREDSSON, L.; HAMMAR, N.; HOGSTEDT, C. Incidence of myocardial infarction and mortality from specific causes among bus drivers in Sweden. International Journal of Epidemiology, Oxford, v. 22, n. 1, p. 57-61, 1993. http://dx.doi.org/10.1093/ije/22.1.57. PMid:8449648.

ASSOCIAÇÃO BRASILEIRA DE NORMAS TÉCNICAS - ABNT. ABNT NBR 15570:2011: transporte especificações técnicas para fabricação de veículos de características urbanas para transporte coletivo de passageiros. São Paulo, 2011.

ASSUNÇÃO, A. A. Condições de saúde e trabalho dos motoristas e cobradores do transporte coletivo das cidades de Belo Horizonte, Betim, e Contagem, MG, Brasil. Belo Horizonte: UFMG, 2013.

BATTISTON, M.; CRUZ, R. M.; HOFFMANN, M. H. Condições de trabalho e saúde de motoristas de transporte coletivo urbano. Estudos de Psicologia Natal, Natal, v. 11, n. 3, p. 333-343, 2006.

BENVEGNÚ, L. A. et al. Prevalência de hipertensão arterial entre motoristas de ônibus em Santa Maria, Rio Grande do Sul. Revista Brasileira de Saúde Ocupacional, São Paulo, v. 33, n. 118, p. 32-39, 2008.

BIGERT, C. et al. Myocardial infarction among professional drivers. Epidemiology, Cambridge, v. 14, n. 3, p. 333-339, 2003. http://dx.doi.org/10.1097/01. EDE.0000057141.91012.80. PMid:12859035.

BJÖR, B. et al. Vibration exposure and myocardial infarction incidence: the VHEEP case-control study. Occupational Medicine, Oxford, v. 56, n. 5, p. 338344, 2006. http://dx.doi.org/10.1093/occmed/kql024. PMid:16717050.

BRASIL. Ministério da Saúde. Representação no Brasil da OPAS/OMS. Doenças relacionadas ao trabalho: manual de procedimentos para os serviços de saúde. Brasília, DF, 2001.

BUHAUG, K.; MOEN, B. E.; IRGENS, A. Upper limb disability in Norwegian workers with hand-arm vibration syndrome. Journal of Occupational Medicine and Toxicology, London, v. 9, n. 9, p. 5, 2014. http:// dx.doi.org/10.1186/1745-6673-9-5. PMid:24517340.

\section{CANADIAN CENTRE FOR OCCUPATIONAL HEALTH} AND SAFETY - CCOHS. Vibration -measurement, control and standards. Hamilton, 2012. Disponível em: <www.ccohs.ca>. Acesso em: 28 out. 2015.

COSTA, L. B. et al. Morbidade declarada e condições de trabalho: o caso dos motoristas de São Paulo e Belo Horizonte. São Paulo em Perspectiva, São Paulo, v. 17, n. 2, p. 54-67, 2003.
EMPRESA DE TRANSPORTES E TRÂNSITO DE BELO HORIZONTE - BHTRANS. Anuário Estatístico. Belo Horizonte, 2009.

FROOM, P. et al. Healthy volunteer effect in industrial workers. Journal of Clinical Epidemiology, Oxford, v. 52, n. 8, p. 731-735, 1999. http://dx.doi.org/10.1016/ S0895-4356(99)00070-0. PMid:10465317.

GUSTAVSSON, P. et al. Myocardial infarction among male bus, taxi, and lorry drivers in middle Sweden. Occupational and Environmental Medicine, London, v. 53, n. 4, p. 235-240, 1996. http://dx.doi.org/10.1136/ oem.53.4.235. PMid:8664960.

IDZIOR-WALUS, B. Coronary risk factors in men occupationally exposed to vibration and noise. European Heart Journal, London, v. 8, n. 10, p. 10401046, 1987.

LEWIS, C. A.; JOHNSON, P. W. Whole-body vibration exposure in metropolitan bus drivers. Occupational Medicine, Oxford, v. 62, n. 7, p. 519-524, 2012. http:// dx.doi.org/10.1093/occmed/kqs096. PMid:22778239.

LIMA-COSTA, M. F.; TURCI, M.; MACINKO, J. A saúde dos adultos em Belo Horizonte. Belo Horizonte: Núcleo de Estudos em Saúde Pública e Envelhecimento; Universidade Federal de Minas Gerais, 2012.

MONSON, R. R. Observations on the healthy worker effect. Journal of Occupational Medicine, Chicago, v. 28, n. 6, p. 425-433, 1986. http:// dx.doi.org/10.1097/00043764-198606000-00009. PMid:3723215.

SOCIEDADE BRASILEIRA DE HIPERTENSÃO - SBH. VI diretrizes brasileiras de hipertensão. Arquivos Brasileiros de Cardiologia, São Paulo, v. 95, p. 1-51, 2010.

THAMSUWAN, O. et al. Whole body vibration exposures in bus drivers: a comparison between a high-floor coach and a low-floor city bus. International Journal of Industrial Ergonomics, Amsterdam, v. 43, n. 1, p. 9-17, 2013. http://dx.doi. org/10.1016/j.ergon.2012.10.003.

VIROKANNAS, H.; TOLONEN, U. Responses of workers exposed to vibration in autonomic nervous function tests. International Archives of Occupational and Environmental Health, Berlin, v. 67, n. 3, p. 201205, 1995. PMid:7591179.

WILLIAMS, B. The year in hypertension. Journal of the American College of Cardiology, New York, v. 55, n. 1, p. 65-73, 2009. http://dx.doi.org/10.1016/j. jacc.2009.08.037. PMid:20117366.

WORLD HEALTH ORGANIZATION - WHO. Physical status: the use and interpretation of anthropometry. Geneva, 1995. 